\title{
Civilisations
}

Revue internationale d'anthropologie et de sciences

humaines

63 | 2014

L'Ancestralité revisitée

\section{De la célébration des ancêtres dans une maison de candomblé de Bahia}

\section{Emmanuelle Kadya Tall}

\section{OpenEdition}

\section{Journals}

Édition électronique

URL : http://journals.openedition.org/civilisations/3682

DOI : 10.4000/civilisations.3682

ISSN : 2032-0442

\section{Éditeur}

Institut de sociologie de l'Université Libre de Bruxelles

\section{Édition imprimée}

Date de publication : 30 septembre 2014

Pagination : 63-79

ISSN : 0009-8140

\section{Référence électronique}

Emmanuelle Kadya Tall, «De la célébration des ancêtres dans une maison de candomblé de Bahia », Civilisations [En ligne], 63 | 2014, mis en ligne le 30 septembre 2018, consulté le 21 avril 2019. URL : http://journals.openedition.org/civilisations/3682 ; DOI : 10.4000/civilisations.3682 


\title{
De la célébration des ancêtres dans une maison \\ de candomblé de Babia
}

\author{
Emmanuelle Kadya TALL
}

Résumé : Le candomblé de Bahia est un culte de possession organisé en nations figurant ainsi des généalogies plurielles qui trouvent leur ancrage en terre brésilienne grâce à l'action conjuguée des dieux africains et des entités autochtones amérindiennes. La description d'un rituel interne dédié aux ancêtres chez un chef de culte bien enraciné dans son époque vient montrer combien ce culte de possession souvent considéré comme un héritage africain, est avant tout une création brésilienne répondant de manière rituelle au mythe de la fraternité raciale né durant la période abolitionniste (1850-1888), mythe auquel Gilberto Freyre donnera ses lettres de noblesse.

Mots-clés : candomblé, Bahia, nations, ancêtres, fraternité, démocratie raciale.

\begin{abstract}
Candomble in Bahia is a possession cult organized into nationalities, thus representing plural genealogies, which are firmly attached on Brazilian soil with the combined help of African gods and indigenous Amerindian spirits. The description of an internal ritual celebrating ancestors in a house where the leader of the cult is truly in tune with the age he lives in, will show how candomble, often considered as an African legacy, is above all a Brazilian creation: Indeed, this possession cult corresponds on a ritual level with the myth of a racial brotherhood born during the abolitionist period (1850-1888), a myth which Gilberto Freyre reinforced in his writings.
\end{abstract}

Keywords: candomblé, Bahia, nationalities, ancestors, brotherhood, racial democracy. 
$\mathrm{D}_{\mathrm{a}}$ ans le candomblé de Bahia, le culte des morts et des ancêtres est distinct du culte des divinités africaines, et rares sont les maisons dédiées spécifiquement au culte des ancêtres. Celles qui en ont la spécialité les honorent au travers des masques de ces fameux Revenants célébrés sur l'ancienne Côte des Esclaves par certains lignages yorubaphones. Il s'agit du culte des Egungun qui semble avoir traversé l'Atlantique avec la fin de l'esclavage pour accompagner les processus de construction identitaire des descendants d'esclaves africains au moment où le Brésil se constituait en Étatnation. Sans pouvoir donner une date précise à l'apparition du culte à Babá Egum ${ }^{1}$, il ne fait aucun doute que son installation sur l'île d'Itaparica, face à la ville de Salvador est due aux échanges intenses entre l'ancienne Côte des Esclaves et Bahia à partir de la fin des années 1830, avec le retour des Retornados en Afrique suite à la plus fameuse révolte d'esclaves à Bahia, la révolte des Malês ${ }^{2}$ survenue en 1835 . L'élite de ces Retournés qui allait participer activement à la traite interlope, allait donc entretenir jusqu'aux années 1930 des relations étroites avec le continent américain. Matory (1999) et Peel (2000) ont montré comment une diaspora africaine christianisée avait au $19^{\text {ème }}$ siècle posé les fondations de l'identité Yoruba, qui du côté brésilien allait devenir un modèle dominant d'africanité à partir du début du $20^{\text {ème }}$ siècle, tandis qu'en Afrique avec la colonisation européenne, l'identité yorouba y devenait le signe de la parfaite assimilation des critères de modernité occidentale avec l'intégration des lois du marché capitaliste et de la religion chrétienne.

Dans cet article, nous ferons peu cas du culte de Babá Egum, car le culte des ancêtres au Brésil est rarement célébré dans un territoire consacré exclusif. Dans la plupart des maisons de candomblé, le culte aux ancêtres participe du cycle liturgique de la maison. Une des rares maisons dédiée strictement au culte de Babá Egum dans la capitale de l'État de Bahia est celle fondée par feu Mestre Didi (1917-2013) artisteplasticien rattaché à une des fameuses maisons mères du candomblé bahianais ${ }^{3}$ (Ilê Axé Opô Afonjá). Cet artiste a ouvert sa maison de prêtre du culte des Egungun, Alapini do Ilê Asipa en 1980 avec l'aide de son épouse, l'anthropologue argentine Juana Elbein dos Santos. Consacré à la divinité de la terre, Obaluayê, avec le titre de Asogba, Mestre Didi a été initié aux Egungun dans un lignage Alapini puis est allé faire sa confirmation comme Balé Xangô à Oyo en 1970, car personne au Brésil n'était capable de l'initier. Les quelques rares maisons de culte consacrées aux ancêtres afro-brésiliens ont été installées dans l'île d'Itaparica, face à Salvador, au début du $20^{\text {ème }}$ siècle si l'on

1 Babá Egum littéralement père Ancêtre, désigne l'ancestralité célébrée au Brésil dans certaines maisons de candomblé affiliées à des lignées Yoruba telle celle des Alapini. Voir l'ouvrage que Julio Braga lui consacre en 1992.

2 João José Reis en a rendu compte dans son célèbre ouvrage revu et augmenté en 2003. Malê désigne les esclaves musulmans, en grande majorité des Hausa selon les recherches menées par cet historien. On attribue généralement à l'influence de ces Malês qui ont complètement disparu du paysage bahianais, le port de vêtements blancs le vendredi, jour dédié au Brésil à la célébration des dieux africains. L'analogie entre le jour dédié aux divinités africaines et celui dédié à la grande prière hebdomadaire en Islam est bien la seule que nous ayons trouvée dans le candomblé.

3 Sa mère biologique était la fameuse mãe Senhora qui a régné dans une des maisons fondatrices du candomblé de Bahia et il a été initié au candomblé par mãe Aninha qu'il considérait comme sa grand-mère. 
considère la profondeur généalogique de ces maisons recensées par Julio Braga ${ }^{4}$. Il est évident que ces maisons fondées loin des rumeurs de la vie urbaine ont participé aux processus de construction d'une identité afro-brésilienne, en permettant aux descendants des anciens esclaves déportés d'Afrique de retrouver une généalogie que la condition d'esclave de leurs ascendants leur avait déniée. Pourtant, si la célébration des dieux africains se décline dans une multitude de nations dans une transformation des nations africaines au sein des confréries catholiques de couleur, le culte des Egungun semble avoir fonctionné, comme aujourd'hui en Afrique, bien plus comme une sorte de société secrète masculine réunissant des hommes en quête de protections mystiques pour le développement de leurs affaires professionnelles que comme un vaste réservoir d'ancêtres pour une population afro-brésilienne en quête de racines.

Le culte des ancêtres dans la plupart des maisons de candomblé participe des rites privés d'une communauté et ne donne pas lieu à des fêtes publiques comme c'est le cas

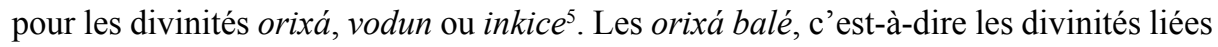
à la mort venues du monde Yoruba entretiennent une relation privilégiée avec Babá Egum dans la plupart des maisons de nation Ketu ou Nagô : on y honore Babá Egum aux côtés des familiers et des affiliations au vaste peuple de saint ${ }^{6}$. Il convient donc de distinguer les maisons consacrées à Babá Egum dont la rareté s'explique par le fait qu'il est un culte consacré aux ancêtres divinisés de certains lignages yoruba, qui a sans doute eu une certaine vogue au début du $20^{\text {ème }}$ siècle, lorsque les relations avec l'ancienne Côte des Esclaves étaient régulières. Au fil du temps, cette quête d'ancestralité s'est réinventée au sein des nations du candomblé et aujourd'hui, comme il a déjà été souligné, très rares sont les maisons dédiées aux mascarades Egungun et ce malgré l'importance symbolique des multiples déclinaisons de la nation yoruba aujourd'hui au Brésil. Dans la plupart des maisons de candomblé, l'ancestralité est activée dans les rituels funéraires (axêxê) effectuées lorsque meurt un leader de la communauté du candomblé. Chaque maison possède son calendrier propre pour commémorer ses morts.

Pour comprendre la place des morts et leur diversité dans le panthéon afrobrésilien, il convient de comprendre le jeu identitaire qui se joue autour de la notion de nation aux Amériques et dans le candomblé. Ainsi, dans un premier temps nous allons montrer comment des attributions catégorielles ethniques héritées de la traite atlantique ont été réinvesties par les descendants d'esclaves et transformées en nations religieuses permettant des affiliations généalogiques plurielles. Dans un second temps, nous décrirons et analyserons comment se décline l'ancestralité dans une maison de candomblé de Bahia où nous avons participé régulièrement pendant cinq ans aux activités rituelles. Cette maison est exemplaire à plusieurs égards : son leader se réclame de la nation la plus afro-centrée, tout en cumulant plusieurs nations dans sa lignée initiatique. Il appartient également à la classe des chefs de culte rénovateurs qui militent pour rendre le candomblé, religion initiatique, accessible au plus grand nombre grâce aux nouvelles techniques de communication. Enfin, tout en ayant rompu avec le

4 Op.cit. 1992.

5 Orixá désigne les divinités originaires de la nation yoruba, vodun celles originaires du monde Fon et inkice celles de l'ancien royaume du Kongo.

6 Traduction littérale de povo de santo qui désigne la vaste communauté des gens du candomblé. 
christianisme depuis de nombreuses années, il a choisi une date du calendrier chrétien pour commémorer les morts de sa maison.

L'analyse des entretiens menés à bâtons rompus avec ce chef de culte ainsi que la description du rite de commémoration des morts viendront témoigner du fait que l'ancestralité s'y inscrit nettement dans un territoire brésilien, faisant ainsi écho à l'hypothèse que nous avons développée dans notre analyse du candomblé de Bahia : loin de célébrer une Afrique lointaine, ce culte hissé au rang de religion en 1985 participe depuis sa naissance à la construction de l'État-nation brésilien aux travers de rituels qui célèbrent dans un même espace-temps un dieu chrétien, des divinités africaines et des entités amérindiennes (Tall 2012).

\section{Le concept de nation dans le contexte de la traite atlantique}

Alors que jusqu'au $16^{\text {ème }}$ siècle, la nation comme forme d'adhésion collective présuppose une communauté de langue, de territoire et de croyance, avec la découverte de l'Amérique et la fracture de la chrétienté, la nation devient synonyme de province, seigneurie, vie civique, bref d'une fidélité à larges rayons (Schaub 2007 : 160).

Avec le développement de la Traite atlantique et l'esprit baroque qui accompagne la Contre-Réforme catholique, les esclaves africains au Brésil vont également être identifiés en termes de nation. Si au début de la Traite, durant le cycle dénommé de Guinée (seconde moitié du $16^{\text {ème }}$ siècle), les attributions catégorielles sont géographiques et très vagues, la Guinée s'étendant en effet sur toute la Côte occidentale de la Gambie au Congo, à partir du cycle d'Angola au $17^{\text {ème }}$ siècle, les attributions ethniques se font plus précises, même si elles sont en grande part inventées par les commerçants du bois d'ébène qui assignent des noms de nations aux esclaves, soit en fonction de leur lieu d'origine supposée, soit en fonction du port par lequel ils vont emprunter le middle passage, ou encore en reprenant les termes utilisés pour les désigner par des populations avoisinantes.

La classification des esclaves en différentes nations africaines par les commerçants d'esclaves procède tout à la fois d'une logique sécuritaire et d'une logique marchande. Dans le premier cas, pour éviter les tentatives insurrectionnelles de plus en plus nombreuses au Brésil avec l'intensification de la Traite. Dans le second cas, parce que cette classification permet de distinguer la marchandise humaine en fonction de certaines qualités : la robustesse pour le negro boçal et la civilité pour le negro ladinho ${ }^{7}$. Cette dichotomie entre l'esclave destiné aux travaux des champs et celui destiné au travail domestique et au petit commerce, si elle semble répercuter la racialisation européenne du $19^{\text {ème }}$ siècle $^{8}$, renvoie également d'abord et surtout - parce que cette classification lui est antérieure, à la culture du goût dans la civilisation européenne. En effet, Gikandi (2011) démontre de manière très convaincante comment l'esclavage agit comme un contrepoint à une culture du goût fondatrice de l'identité européenne moderne au $18^{\text {ème }}$ siècle. L'interaction que Gikandi explore entre culture du goût et

7 Boçal désigne en portugais une personne rustre et ladinho, malin, rusé. On retrouve ces termes également en espagnol.

8 Voir ce que relève Stefania Capone (2000) des stéréotypes raciaux sur les Yoruba et Bantu dans les études afro-brésiliennes. 
esclavage à travers l'analyse de tableaux mettant en scène puissants et esclaves, par exemple, on peut également la retrouver au sein même de la catégorisation des esclaves aux Amériques. En effet, dans l'échelle de valeur qui oppose l'esclave des champs boçal à l'esclave urbain ladinho, il y a la barbarie du premier et la civilité du second, et l'on peut donc dire que même au sein de la catégorie d'esclave, les Européens élaboraient des distinctions basées sur l'opposition barbarie/civilité pour qualifier la valeur marchande des esclaves africains. Lors du cycle Angola (au $17^{\text {ème }}$ siècle) et le cycle de la Côte de Mina et du Golfe du Bénin (18 ${ }^{\text {ème }}$ siècle jusqu'en 1815) la demande en main-d'œuvre esclave augmente pour satisfaire les productions sucrières et caféières des différentes provinces brasiliques ${ }^{9}$ dont Alencastro (2000) dans son ouvrage sur la formation du Brésil dans l'Atlantique Sud souligne le dynamisme et la relative autonomie vis-à-vis de la métropole grâce aux échanges sud/sud favorisés par la navigation atlantique. C'est donc dans cet espace atlantique sud qu'une certaine familiarité ou ressemblance perçue par les marchands d'esclaves entre les États féodaux de l'Afrique de l'Ouest et les empires européens de l'Ancien régime les conduit à distinguer en termes de civilité, des états et royaumes de l'Afrique occidentale et centrale. Cette distinction entre territoires africains aura des répercussions dans la qualification des nations de candomblé jusqu'à aujourd'hui, où la suprématie des cultes originaires de l'ancienne Côte des Esclaves sur ceux originaires de l'ancien royaume Kongo s'exprime en termes de sophistication, de rationalité et d'esthétisme, bref par rapport à des choses qui se rapportent à une culture européenne du goût. Cette culture du goût, loin de disparaître avec les technologies numériques et médiatiques, se trouve alimentée et renforcée avec la circulation des images de rituels, composant de véritables tableaux dont chaque initié, à travers le Brésil et le monde, peut s'inspirer dans sa propre maison. C'est également l'occasion de discuter, de railler et d'alimenter les controverses au sein du candomblé, grâce au fuxico $^{10}$ qui fonctionne comme discours moral.

Aux Amériques, en l'absence outre-mer de garnisons militaires d'importance avant la seconde moitié du $18^{\text {ème }}$ siècle, c'est au clergé qu'était dévolu le rôle de maintenir la loyauté des peuples coloniaux aux Couronnes ibériques. Ainsi, outre les nouveaux Chrétiens ${ }^{11}$ accusés de pactiser avec le Diable, de nombreux esclaves africains soupçonnés indifféremment d'homosexualité et/ou de commerce avec le Diable étaient remis aux autorités judiciaires de l'Inquisition. En Afrique, la prophétesse Kimpa Vita, à l'origine du mouvement messianique des Antoniens, finissait sur le bûcher en 1706 .

L'émergence d'un catholicisme tridentin coïncida dans les territoires coloniaux du Nouveau monde avec la formation d'une nouvelle culture. Le Concile de Trente donna une emphase particulière au clergé séculier et c'est à travers les confréries et associations laïques les plus diverses que l'entreprise missionnaire allait se reconfigurer. Le Christianisme professé par les jésuites et les Ordres mendiants dans les Amériques s'est essentiellement appuyé sur les émotions, la crainte du divin et

9 Nous empruntons le terme à Alencastro (2000) pour distinguer le Brésil colonial du Brésil indépendant à partir de 1822 .

10 Fuxico, littéralement ragot, est très important dans le candomblé et participe de son économie morale, $c f$. J. Braga (1998).

11 Il s'agit principalement des Juifs chassés de la péninsule ibérique qui s'expatrient en Afrique et dans les territoires nouveaux pour échapper aux persécutions de l'Inquisition. 
les représentations dramatisées des différents moments de la vie du Christ. Jésuites et Ordres mendiants ont ainsi été les propagateurs, dans le Nouveau Monde, d'un dogme élaboré pour consolider le pouvoir de l'Église et lutter contre les hérésies ; celui de la transsubstantiation, à travers la célébration de la Fête-Dieu, ou encore la Fête du Divin. Célébrer le corps du Christ et l'ingérer venait renforcer l'adhésion à une communauté des croyants car la parole mangée pour paraphraser Louis Marin (1986) n'est pas sans évoquer d'autres rituels théologico-politiques que le catholicisme de la Contre-Réforme tente de contrecarrer.

La tradition de la confrérie des noirs affranchis et esclaves dévots de Notre-Dame du Rosaire, fondée à Lisbonne entre la fin du $15^{\text {ème }}$ et le début du $16^{\text {ème }}$ siècles, fait remonter la mise en scène des couronnements des rois Kongo à la moitié du $16^{\text {ème }}$ siècle. De nombreux auteurs ${ }^{12}$ se sont intéressés à ces festivités royales Kongo, émettant de nombreuses hypothèses sur une forme de réactivation de cultes et de sociétés secrètes africaines en Europe et dans les Amériques, au sein des confréries et sous le couvert du culte des Saints. Il est cependant très difficile de trouver dans les récits des témoins de ces festivités en Afrique et hors d'Afrique des descriptions suffisamment détaillées pour confirmer pareilles hypothèses. Nous savons bien que dès lors que l'on cherche des correspondances ou des affinités entre l'Afrique et l'Amérique lusophone, on en trouve toujours. Néanmoins, il convient de se poser la question de savoir si ces affinités proviennent d'une culture africaine qui se serait diffusée dans les Amériques, ou d'une culture atlantique née du déploiement de l'Empire portugais dans l'Atlantique Sud. Les historiens ont ainsi noté que l'Inquisition portugaise avait fait plus de victimes dans les concessions et les territoires lusophones qu'en métropole ${ }^{13}$. Au-delà des stratégies d'éviction des négociants étrangers et de civilisation/domestication de territoires lointains, l'on peut aussi comprendre le succès de l'Inquisition et de son imaginaire diabolisant par la crainte de l'Autre, encore inconnu et menaçant. Les récits et hypothèses émises par Lara (2007), loin d'alimenter la théorie bastidienne du masque syncrétique (Bastide 1960), paraissent au contraire favoriser une interprétation en termes d'englobement et de recomposition des pratiques rituelles, dans une logique cumulative que Marc Augé (1982) a bien décrite dans les religions africaines, dont il a souligné le caractère à la fois plastique et totalitaire. Il semble donc assez cohérent que des rituels et célébrations animés par des esclaves africains coïncident avec les grands moments de célébration chrétienne. Non pas tellement pour profiter de la liberté conférée lors des jours Saints que pour s'assurer la bienveillance du Dieu chrétien, dont la force est supérieure à celle des divinités africaines, comme le prouve l'asservissement de leurs adeptes.

Les confréries des esclaves et affranchis étaient aussi qualifiées de confréries des gens de couleur, pour désigner un ensemble regroupant Africains du continent, Africains nés au Brésil (Crioulos), mulâtres et métis. Jusqu'au $18^{\text {ème }}$ siècle, les classifications et hiérarchisations des esclaves africains obéissaient donc à une logique plus générale de mise en ordre de la société coloniale, à laquelle les colons eux-mêmes

12 Voir par exemple, Mello e Souza 2002.

13 Rappelons que l'ancien royaume Kongo a lui aussi été victime des foudres de l'Inquisition à travers la personne de Kimpa Vita dite Dona Beatriz qui a été brûlée vive en 1706, et qui est à l'origine du mouvement messianique des Antoniens dans cette région d'Afrique centrale. 
n'échappaient pas. Si les esclaves étaient d'abord catalogués en termes de nation dès leur port d'embarquement en Afrique, en fonction de leur provenance présumée, ces nations n'ont pris véritablement tout leur sens que dans les Amériques où ces identités ethniques prescrites jouaient un rôle dans la valeur marchande de l'esclave et les types d'asservissements auxquels il allait être soumis.

\section{Les nations du candomblé de Bahia}

C'est au sein des confréries catholiques laïques d'esclaves et d'affranchis que se sont constitués en partie les premiers noyaux qui ont donné naissance aux maisons de candomblé. C'est au cœur même du directoire de ces confréries qu'a mûri une élite sacerdotale dans la seconde moitié du $19^{\text {ème }}$ siècle. L'esclavage aboli, confréries et conseils d'esclaves affranchis se sont délités progressivement au profit de nouveaux espaces religieux organisés à l'instigation de cette élite désireuse d'une véritable autonomie. Les noms de nations comme marqueurs d'identité avaient déjà glissé de l'ethnique vers le religieux, à travers le culte simultané et/ou complémentaire des dieux africains et des saints catholiques. Le culte des saints catholiques continua d'être exercé au cœur des confréries catholiques conjointement avec le culte des orixá ${ }^{14}$ à l'intérieur des maisons de candomblé. Qui plus est, les espaces religieux du candomblé se sont mis sous la protection d'un saint catholique. En effet, beaucoup des anciennes maisons les plus illustres de Salvador continuent d'être gérées par des Sociétés de Bienfaisance sous le patronage d'un saint ${ }^{15}$.

Le candomblé comme institution autonome prend toute son ampleur au cours du $20^{\text {ème }}$ siècle. Les nations y ordonnent les divinités honorées selon leur ancien territoire d'origine. En effet, le culte des vodun(s) et orixá(s) en Afrique a toujours été inscrit dans des territoires socio-politiques nommés et identifiés quel que soit leur itinéraire migratoire ultérieur. C'est ainsi que les divinités Oxum, Ogun et Logunede ${ }^{16}$ sont rattachées par les mythes et légendes à l'ancien royaume de Dassa (Idaxa) ou que la divinité Sakpata a transité de l'actuel Nigéria à l'actuel Bénin par l'ancien royaume de Savalou. Dans un contexte purement liturgique, la nation désigne le territoire d'origine d'une divinité. Les nations de candomblé ordonnent l'ensemble des divinités en fonction du contexte rituel selon une logique hiérarchique d'ancestralité. C'est cette même logique qui intègre comme divinité locale, l'amérindien autochtone sous la figure générique du caboclo. Ancêtre primordial de tous les Brésiliens, l'indien caboclo est le maître de la terre et le premier occupant du sol national. Figure ambiguë, divinité accessible à tous, le caboclo est intégré dans l'univers religieux du candomblé tout en

14 Les orixás en marquant leur origine Nagô/yoruba signalent l'importance des échanges entre l'Ancienne Côte des Esclaves et le Brésil durant la traite interlope.

15 Sociedade Beneficente de São Lazaro pour l'ancienne Vila Flaviana, plus connue aujourd'hui sous le nom du Cuivre (O Cobre) dans le quartier Engenho Velho da Federação, le Centro da Cruz Santa do Axé Opô Afonjá dans le quartier São Gonçalvo do Retiro, ou encore Sociedade Beneficente de São Bartolomeu pour le terreiro Oxumaré situé dans le quartier Vale do Ogunjá.

16 Oxum divinité de l'eau douce, Ogun divinité de la guerre matérialisée dans le fer, Logunede, divinité hermaphrodite chasseur et pêcheur vivant la moitié de l'année dans la forêt et l'autre moitié dans les eaux du fleuve Oxum. 
conservant ses distances. Il est honoré selon le principe d'autochtonie, il fonctionne comme messager et/ou révélateur de désordres sociaux et individuels, et aujourd'hui il compose une nation parmi les autres dans le panthéon afro-bahianais. Le candomblé de caboclo désigne à la fois un espace religieux et une pratique rituelle qui met sur le devant de la scène les divinités autochtones, caboclos, tout en conservant en arrièreplan les divinités majeures du panthéon afro-brésilien.

Dès le début de la fondation des maisons de culte à Bahia, le concept de nation englobe une pluralité de sens où les notions d'antériorité et de pureté participent à la

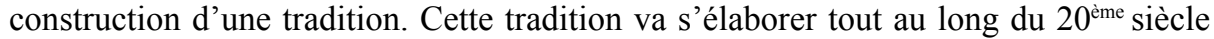
grâce à l'action conjointe de l'élite sacerdotale issue des confréries laïques catholiques et des intellectuels intéressés par la question raciale au Brésil. La tradition du candomblé s'est construite à partir de la fin du $19^{\text {ème }}$ siècle et participe depuis d'un processus de légitimation basée sur une ancienneté (re)trouvée ou (ré)inventée. Les différentes « traditions inventées ${ }^{17}$ » que l'on peut dater du premier quart du $20^{\text {ème }}$ siècle obéissent bien aux mécanismes analysés par Hobsbawm et Ranger (1983) : (a) la nécessité de se référer à l'histoire, fût-elle fictive, pour signifier un continuum nécessaire à la mise en perspective dans le présent et le futur, est particulièrement importante pour des descendants d'esclaves dont la condition statutaire leur interdisait la projection dans le passé, et en conséquence de s'imaginer un futur ; (b) la production de traditions trouve un terrain particulièrement fertile en période de changement social et la fin de l'esclavage en est un particulièrement éprouvant pour une population qui se heurte soudainement à la concurrence sur le marché du travail; (c) l'adaptation de certains modèles anciens dans de nouvelles perspectives.

Des processus de formalisation et de ritualisation extrêmement sophistiqués, établissant des frontières symboliques de plus en plus marquées entre les différentes nations, contribuent à la fixation de la tradition dans le candomblé bahianais. Cependant, ces processus de fixation et de durcissement des traditions, observables depuis le début du $20^{\text {ème }}$ siècle avec une accélération autour des années soixante-dix, loin de marquer une rupture avec l'idéologie dominante marquée par le mythe de la fraternité raciale, en consolident les bases. En effet, les discours sur la pureté, l'authenticité, l'originalité et l'absence de "syncrétisme " dans le candomblé viennent renforcer les signes diacritiques de l'Afrique au Brésil, composant cette mosaïque de nations dont le Brésil moderne se proclame l'héritier.

Aujourd'hui, à Bahia, les nations de candomblé regroupent quatre principaux groupes, (angola, jêje, nagô/ketu et caboclo). Ils se segmentent en de multiples sousgroupes qui s'entrecroisent constamment. Les nations angola, jêje, nagô et/ou ketu ne font plus référence à une origine « ethnico-territoriale », mais à une ou plusieurs lignées symboliques partagées par les membres d'une même communauté/nation religieuse.

Le témoignage de Toluayê, dirigeant d'une maison de candomblé où se célèbrent chaque année, dans la nuit du $1^{\mathrm{er}}$ au 2 novembre les ancêtres du peuple de Saints, en témoigne :

« Je suis de la nation Ketu, ma mère de Saint qui est de Logunede, appartient à la nation Ijexa, parce que Logunede n'existe pas dans l'Alaketu, mais elle a été initiée par

17 Le mythe de fondation d'une des plus anciennes maisons de Salvador ou encore la tradition des douze ministres de Xangô d'une autre maison illustre. 
un père de Saint de la nation Nagô-vodun dans une maison Angola parce qu'il n'avait pas encore ouvert sa propre maison [de candomblé]. ${ }^{18}$

Tout adepte du candomblé se rattache à plusieurs lignées/nations religieuses : celle de sa divinité protectrice, celle de son initiateur, celle de l'espace consacré (axé) où il a été initié, et toutes celles de ses collatéraux et parents en religion.

\section{Célébrer les morts et les ancêtres de la communauté de Toluaye}

Chaque nuit du $1^{\text {er }}$ au 2 novembre, dans le terreiro de Toluayê est organisée une veillée consacrée aux ancêtres de la communauté durant laquelle la frontière entre adeptes initiés et invités est abolie, chacun devant participer activement au rituel en intégrant la ronde qui va jusqu'au petit matin danser en l'honneur des différents ancêtres de la maison.

Mais qui sont les ancêtres qui vont être célébrés durant cette veillée ?

Au cours de cette nuit est commémoré un ensemble pour le moins disparate d'esprits des morts dans la mesure où tous n'ont pas fait l'objet de processus d'ancestralisation. Une multitude de figures est en effet convoquée cette nuit-là : il y a des énergies de la nature, des esprits indigènes et des morts. L'opération de mise en présence, telle que Louis Marin conçoit la représentation, s'effectue de deux manières, l'une réflexive dans un jeu de miroir et l'autre transitive dans sa valeur d'échange. L'évocation ordonnée de divinités africaines selon leur nation, des esprits amérindiens, des morts de la lignée symbolique du leader de la maison et des morts de chaque membre de la communauté, procède, malgré la diversité des figures célébrées, d'un rituel de commémoration de l'ancestralité : l'énumération de morts génériques, de morts généalogiques, c'est-à-dire des morts auxquels les participants initiés sont liés par parenté symbolique (l'initiateur du leader, lui-même relié à un lignage ou des lignages qui le rattachent à une maison fondatrice du candomblé de Bahia, ainsi que chacun de ses adeptes initiés qui peuvent aussi avoir d'autres généalogies), mais également à la parenté biologique de chacun, permettent à tous de chanter différentes catégories de proches disparus et de les transformer en ancêtres bienveillants lors de cette commémoration. Car en effet, ici comme ailleurs, un malheur, une infortune sont fréquemment attribués à l'action d'un mort mécontent, d'un mort errant venant troubler les vivants parce que n'ayant pas reçu les offrandes et les sacrifices lui permettant de se transformer en ancêtre protecteur et bienveillant.

Un des premiers questionnements lors d'une consultation divinatoire concerne les ancêtres du consultant et la première des offrandes sacrificielles leur est toujours adressée. Mais la découverte d'un ancêtre mécontent peut révéler bien des surprises et avant de se rattacher à une lignée « africaine », c'est bien souvent à un autochtone caboclo que l'on se trouve confronté au début d'une quête thérapeutique. Son rôle est primordial dans les pratiques de conversion au candomblé mais il est souvent mis à mal

$18 K e t u$, en référence à l'ancien royaume de Kétou (actuel Bénin). Logunede est une divinité qui est pendant six mois de l'année un jeune chasseur et pendant les autres six mois une belle nymphe qui ne se nourrit que de poisson. Ijexa en référence à l'ancien royaume Ijexa dont la capitale était Dassa (actuel Bénin). Nagô-vodun littéralement divinité nagô, i.e d'origine yorouba. Nagô désigne les yoroubaphones du Bénin. Angola en référence à l'ancien royaume Angola (situé entre les actuels États du Congo et de l'Angola). 
par les idéologues afro-centristes qui pensent qu'intégrer le caboclo dans leurs discours et leurs pratiques revient à dénaturer l'africanité de leur religion. C'est pour cette raison que Toluayê, le leader dont nous avons accompagné cinq ans durant la majorité des rituels publics ou restreints à sa communauté d'adeptes, tout en nous conviant dès notre première rencontre à la célébration des ancêtres chez lui, nous dévoilait seulement trois ans plus tard, l'importance du caboclo dans la fondation de sa maison de culte. Son ancêtre le plus perturbateur et à l'origine des troubles mentaux qui l'avaient affecté dès son plus jeune âge, loin d'être un lointain parent africain, était un esprit amérindien qu'il lui avait fallu installer dans son terreiro, après avoir fait de nombreuses recherches ${ }^{19}$. Depuis, il est célébré le dernier samedi du mois de novembre pour y clore les festivités liées à l'ancestralité.

Ainsi, comme beaucoup de ses confrères venus au candomblé par la maladie ou l'infortune, Toluayê a commencé sa carrière de leader religieux et thérapeute en organisant des sessions de caboclos. Contrairement aux divinités africaines peu causantes, les caboclos sont des esprits amérindiens doués de clairvoyance et lorsqu'ils sont appelés et qu'ils descendent dans le corps des médiums, ils répondent en toute simplicité aux questions qu'on leur pose. Ils mettent également en garde les uns et les autres contre certains de leurs agissements et n'hésitent pas à se montrer parfois violents. Craints et respectés, les caboclos sont des entités très proches des êtres humains avec lesquels ils partagent un grand nombre de traits de caractère. Si la plupart des leaders des maisons liées aux nations africaines les plus afro-centrées de Bahia taisent leurs liens avec les caboclos, dès qu'on se penche sur leur itinéraire religieux, à la rare exception de certains de ceux qui ont hérité d'une charge, le caboclo y a été la première figure rencontrée.

Toluayê possède également un Babá Egum en raison de son affiliation à la nation Ketu. Maître de la terre, sa divinité principale Obaluayê est un orixa balé eku, c'est-àdire un dieu lié à la mort puisque c'est la terre qui accueille les défunts avant que ces derniers n'atteignent les différents ciels ${ }^{20}$. Ce n'est pas son initiatrice qui lui a installé Babá Egum mais un spécialiste des Egungun résidant sur l'île d'Itaparica à Ponte de Aréia. En général, on installe Babá Egum bien après avoir conclu son initiation et ouvert sa propre maison de culte. Cependant les leaders dont la divinité supérieure est liée à la mort doivent l'installer le plus rapidement possible. Toluayê a installé le sien deux ans après avoir reçu le deká ${ }^{21}$ et c'est sur le littoral nord qu'il en a retrouvé les traces $^{22}$ grâce au jeu divinatoire du spécialiste consulté. Son ancêtre « yoruba » est un vieil esclave noir de haute taille, si l'on en croit la description faite par une inconnue qui est venue frapper à la porte du terreiro, assurant qu'elle avait été conviée par le maitre

19 On taira les procédés employés pour l'installer et son nom par respect pour notre hôte car tout ce qui touche à l'ancestralité participe de l'intimité la plus profonde de l'individu.

20 C'est Oyá la déesse du vent et des tempêtes qui est la messagère permettant d'accéder aux neuf ciels ou orun.

21 Le deká est l'ensemble des objets que le novice reçoit à la fin de son initiation, dont le couteau qui a été utilisé pour lui raser la tête et qui vont être enterrés au centre du barracão avec la pierre et les offrandes animales et végétales qui accompagnent le rite de fondation d'une maison de candomblé. 
de maison, le jour où ce dernier, qui ne connaissait pas cette femme, était justement en train de procéder au rituel d'installation de cet ancêtre.

La commémoration des ancêtres commence habituellement en début de soirée entre 19 et 20 heures. Avant cela, le personnel initié de la maison a disposé au centre de la salle consacrée (barracão) à la descente publique des dieux, de petites assiettes et coupelles blanches contenant les différents mets destinés aux ancêtres avec aux deux extrémités faisant face à l'entrée, deux têtes de cire, l'une masculine, l'autre féminine. À l'intérieur de ces têtes sont entreposés les restes du Babá Egum. Deux magnifiques bouquets de chrysanthème blanc et de lys ornementent l'espace du pilier central qui est le lieu où est enterré la force axé de la maison. C'est autour du pilier, invisible chez Toluayê, mais marqué par des carreaux noirs en forme de croix au centre de la salle, que les rondes aux ancêtres vont se succéder tout au long de la nuit. Ce pilier sous lequel réside la force axé, est en correspondance avec le faîte du barracão, nommé cumeeira (crête, partie la plus haute du toît) qui active les énergies entre la terre et les ciels (orun) de la divinité leader de la maison. Chez Toluayê, en raison de la jeunesse du terreiro, il y a homologie entre la divinité de la maison et celle du leader.

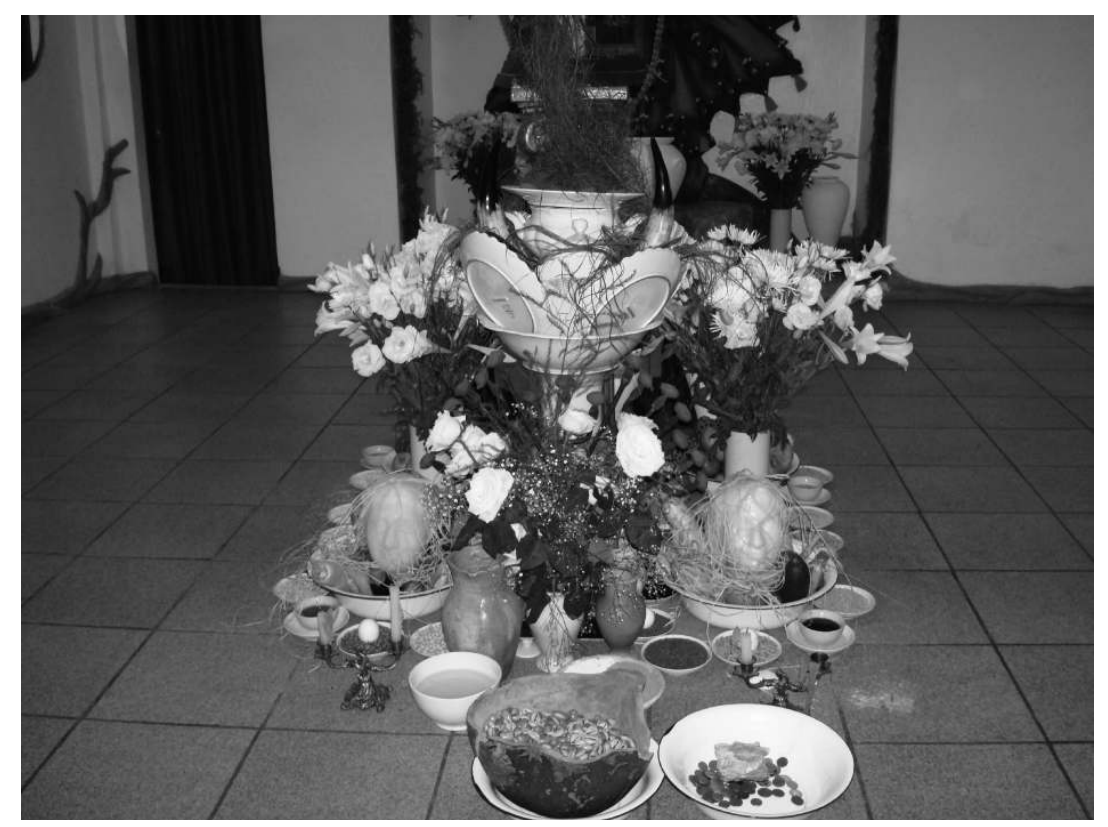

Figure 1: Offrandes de nourritures végétales aux ancêtres dans le barracão. (C) Kadya Tall, 2010.

Chacun arrive au terreiro les bras chargés de gourmandises, car à cette occasion, on mange tout au long de la nuit des petits fours et des gâteaux : il s'agit, comme lors des fêtes anniversaires dont les friandises sont les mêmes, de célébrer dans la joie et la bonne humeur les morts de chacun qui cette nuit-là sont de tous. Les boissons, uniquement sucrées, sont fournies par la maison, c'est-à-dire par le leader et les initiés. Ce jour-là, 
les initiés et profanes sont tous entièrement vêtus de blanc, les femmes en jupe de coton ample, blouse, fichu et écharpe, les hommes en chemise ou t-shirt et pantalon. Avant de débuter la ronde et les chants, chacun passe à la séance de maquillage qui consiste à se faire enduire les tempes, les paupières et le creux des poignets de poudre de kaolin parfumé (efun), avec pour les hommes, un fil végétal ${ }^{23}$ enserré au poignet gauche, et pour les femmes, le fil ceint autour du fichu dont elles se recouvrent la tête. Ces fils de raphia servent à éloigner les mauvais morts. Le maquillage se déroule dans une atmosphère bon enfant qui diffère avec les préparatifs habituels d'une fête publique. Il n'y a pas de défi ce soir-là, sinon celui de montrer son respect pour les ancêtres en dansant avec entrain. Après le maquillage, chacun s'empresse auprès d'une matrone pour troquer des piécettes ou des petits billets contre des cauris qui serviront à chacun à racheter sa vie au cours de la ronde qui va s'étirer jusqu'aux premières heures du matin. Chacun à tour de rôle prendra la place de caissier au cours de la nuit, en se plaçant à l'instar des musiciens, au plus près du centre du barracão. Le rythme de la ronde s'accélère à la fin pour permettre à chacun de réceptionner les cauris de vie.

Dans la journée précédant la veillée, des sacrifices animaux ont été effectués pour les différentes divinités de la communauté. C'est Oyá, la déesse des vents et de la tempête qui est l'ordonnatrice des rites de commémoration aux ancêtres en raison de son rôle de médiatrice entre les vivants et les morts. Après les sacrifices de coqs au messager Exu, une chèvre, une poule, une pintade et une colombe lui sont consacrées. Les dieux et les ancêtres mâles ont reçu un mouton, des coqs, des pigeons, des pintades mâles et des canards. Avant l'aube, les entrailles et les parties non consommables des sacrifices seront jetées dans une brousse et le lendemain de la veillée, une partie des animaux sacrifiés sera préparée pour le repas communiel qui clôt ce rituel pour les initiés de la maison. Cependant, la clôture finale des rites dédiés aux ancêtres advient chaque année le dernier samedi du mois avec la célébration de l'ancêtre caboclo du leader de la maison. Chez Toluayê, le mois de novembre dans sa totalité est dédié à la célébration des ancêtres et aucune fête de sortie d'initié ou de célébration d'un dieu n'est programmée durant cette période. Les rituels d'ancestralisation (axêxê) s'accomplissent à cette époque de l'année. Sont concernés les parents des lignages d'initiation et les parents biologiques du leader de la maison. Ceux-ci bénéficient de rites spécifiques qui peuvent débuter lors de la veillée qui ouvre en quelque sorte le cycle de célébration de la mort et de l'ancestralité, mais qui se prolongera au-delà de cette nuit commémorant l'ensemble des ancêtres des personnes présentes. Cette nuit-là tout un chacun est acteur et l'unique public jugeant la performance du groupe sont les ancêtres honorés.

La séance de maquillage terminée, Toluayê bat le rappel et chacun pénètre dans le barracão/salon de danse en se regroupant par genre et par ordre hiérarchique, pour composer deux rondes, la première proche du cercle constituée des hommes conduits par Toluayê, la seconde conduite par une initiée non médium (ekede). Habituellement, hommes et femmes se mélangent dans les rondes souvent au nombre de trois (membres de la hiérarchie- initiés- novices), mais cette nuit-là, sans doute en raison de la présence de profanes, on respecte la division des sexes rarement observée dans les maisons de candomblé modernes. 
Après l'offrande à $E x u^{24}$ au seuil du barracão, la porte est soigneusement refermée et elle ne sera pas rouverte de la nuit car cette commémoration n'est pas publique contrairement aux fêtes dédiées à la consécration des dieux et de leurs montures. Toluayê fait tonner de la poudre de fusil puis souffle de la poudre d'efun sur chacun des participants. Ensuite il fait une courte déclaration pour expliquer le sens de la commémoration : « cette veillée est pour célébrer les ancêtres de tous, des Blancs, des Noirs et des Métis car l'humanité n'est qu'une. Ce n'est pas une fête triste, au contraire, elle doit être très gaie car c'est la mort qui fait la vie et de toute façon, on ne meurt qu'une fois ».

Le xiré, c'est-à-dire l'ordonnancement des chants dédiés aux divinités obéit ce soir là à deux logiques, celle de séniorité et celle de préséance. Habituellement le xiré commence par les divinités les plus jeunes pour terminer par les plus anciennes. Or la logique qui prime en cette veillée, c'est la logique historique couplée à la question de l'autochtonie. C'est ainsi que les premiers chants sont adressés aux divinités Angola et à certaines figures du panthéon des caboclos très souvent convoquées dans les candomblés de nation Angola/Kongo. Un entracte d'une demi-heure qui sera le seul de la soirée permet à chacun de se restaurer. Il permet également de marquer une frontière entre les différents types d'ancêtre qui sont commémorés cette nuit-là. En effet, après les ancêtres les plus anciens, viennent ceux de la maison qui est rappelons-le de nation Ketu : sont alors évoqués vodun du Danxomé et orixá de l'ancien royaume de Ketu. Au fur et à mesure de l'avancée de la nuit, les transes se font de plus en plus nombreuses chez les médiums possédés par des divinités liées à la mort. Cependant, personne ne dansera pour Babá Egum ce soir-là. Il n'apparaît que lors des fêtes anniversaires, de sept en sept ans, et c'est le maître de céans qui l'incorpore. Après les chants invoquant les divinités de la nation Jêje-Nagô ${ }^{25}$, Toulayê reprend la parole pour louer les morts récents, les parents vivants et morts de chacun et les membres prééminents de sa lignée généalogique qui est, rappelons le, à plusieurs branches puisqu'il est tout à la fois relié aux nations Angola, Jêje-Nagô et Caboclo.

Cette nuit-là, les trois musiciens ont délaissé leurs tambours atabaque et la scène qui leur est habituellement réservée dans une sorte d'alcôve, pour se placer au plus près du cœur du barracão, en son centre, en jouant de deux tambours zenli ${ }^{26}$ et du plus petit des tambours atabaque sur lequel on frappe avec des baguettes les rythmes Angola.

Toluayê lance les chants, mais ça peut également être un $\operatorname{og} \tilde{a}^{27}$, chants qui sont repris en chœur par l'ensemble des présents. De temps à autre, Toluayê consulte un grand cahier noir gardé dans l'enceinte des ancêtres et des caboclos qui se situe à l'entrée du barracão, dans un espace semi-ouvert, car le caboclo est un esprit de la forêt. Dans les maisons traditionalistes, les esprits amérindiens ne sont jamais honorés dans l'enceinte du barracão, mais en dehors, au pied d'un arbre le plus souvent. Cependant, il est

24 Le messager trublion mais également divinité des carrefours, intermédiaire des dieux et des hommes.

25 Jêje-nagô désigne la nation des derniers esclaves africains arrivés de l'ancien Danxomé lors de la traite interlope, regroupant des Fon et des Yoruba.

26 Le zenli est un tambour en terre cuite frappé d'une lanière de cuir. Il est utilisé comme en pays Fon au Bénin, dans les rituels funéraires du candomblé de nation Jêje- Nagô au Brésil.

27 Titre désignant un homme initié qui n'est pas possédé par ses divinités. 
difficile de considérer le caboclo comme un être incongru ou un élément syncrétique dans le panthéon bahianais dans la mesure où, tant dans les maisons de nation Angola ou Ketu/Yoruba, l'arbre en question (en général un fromager) est nommé Loko $^{28}$ dans la nation Nagô/Ketu/Yoruba et Tempo dans la nation Angola. Le caboclo comme figure annonciatrice et médiatrice est absolument nécessaire à toute pratique rituelle dans le candomblé et la séparation qu'on pourrait considérer comme artificielle entre caboclos et orixás vient juste fixer les places de chacun.

Chez Toluayê, la forêt des caboclos et des ancêtres se résume à quelques ficus et un pied de fruits de la passion au-dessous desquels sont entreposés les restes de l'ancêtre amérindien, les poteries individuelles de chaque initié de la maison et l'autel d'Ogun, la divinité du fer qui ouvre les chemins. Babá Egum quant à lui réside dans la chambre d'Oyá.

Contrairement aux fêtes publiques où les acteurs et l'auditoire sont séparés, ce dernier assis dans l'espace réservé aux ancêtres et caboclos et sur une sorte d'estrade qui domine le salon/barracão où initiés, novices et divinités se côtoient et fusionnent parfois, lors de la veillée aux ancêtres, la frontière entre profane et sacré s'estompe, pour signifier que tous participent en toute équité à la célébration de ce que tout être humain libre et du commun possède, à savoir des ancêtres. Épreuve de bravoure s'il en est, voire de résilience pour un culte dont les premiers créateurs appartenaient à la catégorie des esclaves dont on sait que leur condition les mettait hors d'accès à la généalogie et l'ancestralité. C'est peut-être la raison pour laquelle lors de cette soirée, les frontières entre initiés et profanes sont abolies comme pour signifier combien l'ancestralité est/ doit être accessible à tous.

Lors de cette veillée, la puissance des ancêtres ne tient pas au pouvoir de représentation, sur une scène théâtrale - le barracão - que les médiums et les membres de la hiérarchie du culte mettent en image, dans une présentification des divinités invoquées pour un public qui, par son regard, en légitime le pouvoir. Il ne s'agit pas d'une succession de portraits où le leader du culte serait tout à la fois le peintre et le cadre d'un tableau-portrait ${ }^{29}$. Cette nuit-là, profanes, novices et initiés participent sans distinction à cette commémoration en dansant en ronde autour du pilier central, entonnant en chœur les chants lancés par le chef de culte ou un de ses adjoints.

Que pouvons-nous retenir de cette veillée ? Tout d'abord, la date de la nuit du $1^{\mathrm{er}} \mathrm{au}$ 2 novembre indique la contiguiité entre la célébration de figures saintes et/ou divines et de morts appartenant au personnel liturgique et à ceux de chacun des participants à la veillée : Saints catholiques, divinités africaines, entités amérindiennes et élite sacerdotale y sont célébrés à l'unisson dans une logique historique où les autochtones caboclos et les premiers africains venus du pays Kongo (et aujourd'hui de nation Angola) arrivés dans les territoires du Nouveau monde reçoivent les premières louanges.

Cependant, la centralité de la nation Ketu chez Toluayê se donne à voir dans les deux têtes de cire qui encadrent les offrandes de nourritures sèches au centre du salon/ barracão et on peut dire qu'il y a cette nuit-là, homologie entre Babá Egum présentifié

28 Loko, terme fon pour désigner l'iroko, l'arbre sous lequel est enterré le placenta du nouveau-né dans toutes les maisons du Bénin méridional (Fon-Nagô-Gun).

29 Voir Tall 2014, où l'auteure s'inspire de la théorie marinienne du pouvoir dans sa représentation pour rendre compte de la diversité du rayon d'action d'un chef de culte dans le Bénin contemporain. 
par ces deux têtes contenant ses restes et Toluayê, le leader de la maison, dans une présence-absence signifiée par leur emplacement au-dessous du faîte de la maison. En effet, si Babá Egum est célébré tous les sept ans, il n'en demeure pas moins que sa présence est annuellement notifiée, tout comme l'est l'ensemble des ancêtres de la communauté et de son leader. En effet, les morts de la famille biologique, les morts de la famille religieuse déclinées en de multiples nations, les divinités de la nature, africaines et amérindiennes qui sont chantées tout au long de la nuit sont mis en présence à travers chants, musiques, danses et transes dans un cadre, ici l'espace-temps du calendrier chrétien, même si cet espace temps s'allonge sur un mois entier.

En outre, si l'activité rituelle qui se déploie autour de la célébration des ancêtres nécessite en amont et en aval le sacrifice et le sang animal, la veillée célèbre moins la vie opposée à la mort que la reproduction induite par la mort, c'est-à-dire la naissance qui peut être également souvent perçue à la deuxième génération comme une renaissance d'un ancêtre : ainsi, Godelier (2014) a-t-il rappelé récemment combien partout, la naissance est l'inverse complémentaire de la mort. On comprend alors pourquoi ce sont des friandises qui sont servies cette nuit-là, comme lors de l'anniversaire d'un an de tout nouveau-né au Brésil.

Mais si durant la veillée, sucreries et gourmandises composent ce que chacun va ingérer au long de la soirée qui s'étire jusqu'aux premières heures du matin, cette partie du rite ne serait pas complète si elle n'était pas insérée dans un temps plus long dans lequel des processus de transsubstantiation viennent réitérer la rencontre/présence des ancêtres et de leurs descendants : en effet, pour régénérer la puissance des ancêtres, des nourritures sèches ou sucrées ne suffisent pas à alimenter le corps des dieux : comme dans tous les rituels du complexe abrahamique, la consommation de chair animale permet de faire se rencontrer et fusionner différentes catégories d'êtres et par là augmenter la puissance de chacun. Contrairement à René Girard (1972) qui pensait que le rituel chrétien grâce au rituel eucharistique mettait fin au rituel sacrificiel, nous pensons au contraire, que loin d'être symbolique, le rituel eucharistique démultiplie les épreuves mystiques à travers l'ingestion de l'hostie et l'expérience de la transe, du moins dans la pratique des adeptes du candomblé ou du Christianisme populaire dans les Amériques ibériques. En effet, l'expérience de l'eucharistie n'y est nullement considérée comme quelque chose de symbolique mais comme une expérience qui permet de fusionner avec le divin. Loin d'éloigner le croyant de l'objet de son adoration, les processus de transsubstantiation permettent au contraire de faire corps avec lui : cela s'expérimente à travers les transes de possession et la consommation collective des animaux sacrifiés en offrandes à des divinités particulières. Ce ne sont pas des gestes anodins et lors des transes, il est possible d'identifier les expériences fusionnelles grâce aux danses et musiques des divinités invoquées. Dans la confusion des genres (animaux, humains, divins), ce qui permet de les identifier, ce ne sont pas seulement des images mais les sons et les danses qui les accompagnent.

Enfin, contrairement à d'autres rituels du candomblé qu'il nous a été donné d'observer chez Toluayê ou ailleurs, lors de la commémoration des ancêtres, la contiguïté entre figures chrétiennes et païennes n'est pas immédiate. Elle est simplement suggérée par l'espace temporel dans lequel se déroule le rituel, on pourrait considérer cet espace comme le cadre du tableau qui lui donne tout son sens. Mais de quel sens s'agit-il ? Celui de redonner à chacun une ancestralité, en veillant à ne pas hiérarchiser initiés 
et profanes, ancêtres amérindiens, africains et européens. Si la séparation de genre et l'ordre hiérarchique sont respectés plus que de coutume dans cette maison résolument moderne, l'absence de frontière entre personnes profanes et personnes initiées ainsi que l'absence de rituel sacrificiel durant la longue veillée semble vouloir mettre en exergue, comme un acte militant, l'ancestralité de chacun, dans sa multiplicité et singularité. Acte d'autant militant lorsqu'on considère l'histoire du candomblé né tout à la fois comme un mouvement de contestation d'un ordre esclavagiste au sein des confréries catholiques laïques de couleur et comme un mouvement identitaire dans le cadre de l'idéologie de la cordialité raciale.

Ainsi faire de la célébration des ancêtres un rituel ouvert aux personnes profanes, mais proches de la communauté d'initiés, vient renforcer la valeur de l'ancestralité pour chacun et si les rituels sacrificiels réservés aux initiés se déroulent avant et après la veillée semi-publique, il n'en demeure pas moins qu'ils participent à l'effectuation du pouvoir de représentation. En effet, les processus de transsubstantiation à l'œuvre lors des sacrifices animaux viennent renforcer la communauté des adeptes, la manducation des corps sacrifiés et l'expérience de la descente des dieux dans le corps des médiums transformant adeptes, animaux sacrifiés et spectateurs en acteurs dont les images se reflètent et se confondent comme dans un miroir.

En guise de conclusion, on peut retenir le caractère pluriel des ancêtres invoqués lors de la veillée du $1^{\mathrm{er}}$ au 2 novembre. Ce caractère pluriel ne tient pas seulement à la diversité des personnes qui participent au rituel ce soir-là. En effet, rappelons que dans n'importe quelle maison de candomblé, indépendamment de l'appartenance sociale du maître ou de la maîtresse des lieux, il y a toujours une mixité sociale et raciale dans ces communautés. Cette mixité qui n'exclut pas des rapports sociaux très hiérarchisés, parfois inversés dans l'espace sacré du terreiro, n'est pas le fait du hasard. Elle est en effet toujours recherchée par les membres supérieurs de la maison de candomblé afin de rendre compte de la diversité ethnico-raciale du Brésil, comme s'il s'agissait de mettre en œuvre le mythe de la fraternité raciale et de la démocratie ethnique telle qu'il a été mis au pinacle par Gilberto Freyre ${ }^{30}$.

\section{Références citées}

Alencastro, Luis Felipe, 2000. O Trato dos Viventes. Formação do Brasil no Atlântico Sul. São Paulo : Companhia das Letras.

Augé, Marc, 1982. Génie du paganisme. Paris : Gallimard.

BAstide, Roger, 1960. Les religions africaines au Brésil. Paris : PUF.

Braga, Julio, 1992. Ancestralidade afro-brasileira : O culto de Babá Egum. Salvador : CEAO/Ianamá.

-, 1998. Fuxico de candomblé. Feira de Santana : Universidade Estadual de Feira de Santana.

CAPONE, Stefania, 2000. «Entre Yoruba et Bantou : l'influence des stéréotypes raciaux dans les études afroaméricaines », Cahiers d'Études africaines XL (1), 157, pp. 55-77.

30 Pour plus de détail sur cette idéologie et son histoire voir Guimarães (2002). 
Freyre, Gilberto, 1974. Maîtres et esclaves. La formation de la société brésilienne. Paris : Gallimard.

GiKand, Simon, 2011. Slavery and the Culture of Taste. Princeton : Princeton University Press.

GIRARD, René, 1972. La violence et le sacré. Paris : Grasset.

Godelier, Maurice (éd.), 2014. La mort et ses Au-delà. Paris : CNRS éditions, « Bibliothèque de l'anthropologie ».

Guimarães, Antônio Sergio, 2002. « Démocratie raciale », Cahiers du Brésil contemporain, 49-50, pp. 11-37.

Hobsbawm, Eric, et Terence Ranger (éds), 1983. The Invention of Tradition. Cambridge/New York : Cambridge University Press.

LARA, Silvia, Hunold, 2007. « Une fête pour les historiens. La relation de Francisco Calmon (1762) et la figure du Roi du Congo dans le Brésil colonial », Cahiers du Brésil Contemporain, Paris, Maison des Sciences de 1'Homme, 65-66, pp.17-57.

MARIN, Louis, 1986. « Le corps glorieux du Roi », in L. Marin, La parole mangée et autres essais théologicopolitiques. Paris : Méridiens Klincksieck/Montreal : Boréal.

-, 1994. De la représentation. Paris : Gallimard.

-, 2006. Opacité de la peinture. Paris : Editions de l'EHESS.

MatoRy, J. Lorand, 1999. « The English Professors of Brazil : On the Diasporic Roots of the Yorùbá Nation », Comparative Studies in Society and History, 41, pp. 72-103.

Mello e Souza, de, Marina, 2002. Reis negros no Brasil escravista. História da Festa de Coroação de Rei Congo. Belo Horizonte : Editions UFMG.

Peel, John, D.Y., 2000. Religious Encounter and the Making of the Yoruba. Bloomington : Indiana University Press.

ReIS, João José, 1988. « Magia jeje na Bahia : a invasão do calundu do Pasto de Cachoiera, 1785 », Revista brasileira de História (São Paulo), 8 (16), pp. 57-81.

-, 1991. A morte é uma festa, ritos fúnebres e revolta popular no Brasil do século XIX. São Paulo : Companhia das Letras.

-, 2003. Rebelião Escrava no Brasil. A História do Levante dos Malês em 1835, ed. revista e ampliada. São Paulo : Companhia das Letras.

Schaub, J.F., 2007. « Le sentiment national est-il une catégorie pertinente pour comprendre les adhésions et les conflits sous l'Ancien Régime? », in A. Tallon, Le sentiment national dans l'Europe méridionale au XVI ${ }^{\text {eme }}$ et XVII ème siècles, pp. 155-167. Madrid : Casa de Velázquez, 97.

TALl, Emmanuelle, Kadya, 2012. Le candomblé de Bahia miroir baroque des mélancolies postcoloniales, préface de Pierre-Antoine Fabre. Paris : Editions du Cerf, coll. Sciences sociales et religion.

-, 2014. « On Representation and Power : Portrait of a Vodun Leader in Present-Day Benin », Africa, 84 (2), pp. 246-268.

Vieira Nascimento, Ana Amélia, 2000. « As Irmandades na Bahia », Catálogo das Irmandades, Ordens Terceiras e Confrarias, Documentos do Arquivo Público do Estado da Bahia, Salvador, pp. 11-15. 